\title{
Random-error minimization during cross-correlation of early-type spectra
}

\author{
W. Verschueren ${ }^{\star}$ and M. David \\ Astrophysics Research Group, University of Antwerp (RUCA), Groenenborgerlaan 171, B-2020 Antwerpen, Belgium
}

Received October 16, 1998; accepted February 18, 1999

\begin{abstract}
Owing to the typical morphology of spectra of early-type stars, random errors play a much more important role in the derivation of their radial velocities than in the case of late-type spectra. We derive a generalised lower bound of the random error on a cross-correlation derived radial velocity shift in the presence of random noise on both object and template spectrum, and discuss its dependence on spectral parameters and noise. In order to limit in practice the random error to this lower bound for earlytype spectra, we show that a number of specific crosscorrelation peak centering techniques are required. The influence of rotational mismatch between object and template on the random error is examined. Finally, the widely used error estimate based on the so-called $r$-statistic is critically evaluated in the context of early-type spectra.
\end{abstract}

Key words: methods: numerical — techniques: radial velocities - techniques: spectroscopic — stars: early-type

\section{Introduction}

Already in mid-century, the technique of cross-correlation was known in the field of signal-processing as it was implemented in radar-receivers to measure time-delay (distance). A rigorous discussion of the precision of such measurements was given by Woodward \& Davies (1950) (see also Woodward 1953). Based on these results a similar technique for the measurement of Doppler shifts in astronomy was proposed by Fellgett (1953) and developed in practice by Griffin (1967). Since then it has been widely used and discussed in the astronomical literature (see e.g. Tonry \& Davis 1979; Connes 1985 and references therein). A major turning point was the advent of

Send offprint requests to: W. Verschueren,

e-mail: verschue@ruca.ua.ac.be

* Postdoctoral Fellow of the Fund for Scientific Research Flanders (Belgium) (F.W.O.). digitized spectra (and computers to handle them) which made it possible to represent a spectrum as a function of $\log$ (wavelength) so that a stellar Doppler-shift measurement became the perfect analogue of the measurement of time-delay in radar. Significant differences with the case of signal-processing in radar are that the "signal" in our case is not an isolated pulse, and that in general a variation of the noise level with wavelength has to be taken into account (see also Fellgett 1953).

Cross-correlation techniques for the derivation of stellar radial velocities have so far been applied most successfully to late-type spectra. These contain numerous, sharp, and useful lines which guarantee a narrow well-defined cross-correlation peak whose centering is straightforward (e.g. Scarfe et al. 1990; Latham 1992; Baranne et al. 1996). Precisions (random errors) and accuracies (systematic errors) in the range $0.1-1 \mathrm{~km} \mathrm{~s}^{-1}$ are routinely obtained from these spectra. The situation for early-type spectra, however, is different at three levels. First, owing to the low line density and typically high rotational velocity, the random error on a radial velocity shift is much higher for a given signal-to-noise and a given wavelength span of the spectra. Secondly, the cross-correlation peak in general is much broader due to the occurrence of intrinsically broad lines ( $\mathrm{H}$ and $\mathrm{He})$ and due to rotation; in addition, it contains important sub-structure caused by the mixing of spectral lines of different width; both complicate its accurate centering. Figure 1 shows an example of the differences occurring between early and late-type spectra. Thirdly, also the accuracy of the measured radial velocity shift is lower due to, on the one hand, a much more likely (and more harmful) spectral mismatch between object and template, and on the other hand, the occurrence of spectral lines influenced by atmospheric velocity fields. A more detailed discussion of the problems related to radial velocity work on early-type stars can be found in Verschueren (1995). It is important to realise, however, that some of the problems just mentioned are also likely to occur for late-type spectra if a precision and accuracy one or two 
orders of magnitude higher would be aimed at (e.g. due to convective shifts).

Improving the precision and accuracy of radial velocities of early-type stars as much as possible (in particular getting below the $1 \mathrm{~km} \mathrm{~s}^{-1}$ level) is important for a variety of astrophysical applications, such as kinematical and dynamical studies of young stellar groups, detection of earlytype binaries, and correction of high-precision proper motions for perspective acceleration. The first results of a study to improve the accuracy for early-type stars by minimizing spectral-type mismatch is presented elsewhere (Verschueren et al. 1999a,b). Here, we address the minimization of random errors (due to photon and read-out noise) in the absence of any intrinsic object-template spectral mismatch; this is related to the problems of the first and second level mentioned above.

In Sect. 2, we derive a theoretical lower bound to the random error on a radial velocity shift, taking into account random noise on both object and template spectrum. Section 3 shows which specific cross-correlation function fitting techniques are required to minimize the random error in practice, while Sect. 4 discusses the influence of rotational mismatch on the random error attained. Section 5 deals with the estimation of the actual random error on a measured Doppler shift, i.e. taking account of the actual way of extracting the information. Section 6 lists the conclusions.

\section{Noise-limited precision}

Random errors set a theoretical upper limit to the radial velocity precision attainable from a given spectrum. Quantifications of this minimum error as a function of the intrinsic morphology of the spectrum and of its random errors were computed by several authors (Connes 1985; Merline 1985; Brown 1989; Butler et al. 1996). These results all assume that the observed spectrum is compared with a noise-free reference spectrum. The latter is of course not the case when cross-correlating with observed templates. In addition, many different templates are required to closely match the different spectral types when dealing with early-type spectra, which makes it more difficult to acquire all of them with a very high $S / N$. We therefore derived a generalised expression for the minimum random error on a cross-correlation shift in the presence of random errors on both object and template. In order to be able to consider sub-pixel errors, we assume throughout that the spectra are oversampled which is a condition generally met for early-type spectra at intermediate and high resolution (see also Sect. 3 ).

Assume an object spectrum $S_{\mathrm{O}}(x)$ and an intrinsically identical template spectrum $S_{\mathrm{T}}(x)$ which have been sampled (i.e. integrated) in $N$ pixels with constant pixel-size $\Delta x$ (the $x$-coordinate may in general be any function of wavelength $\lambda$ ). We assume both spectra to be multiplicatively normalised to an identical but otherwise arbitrary
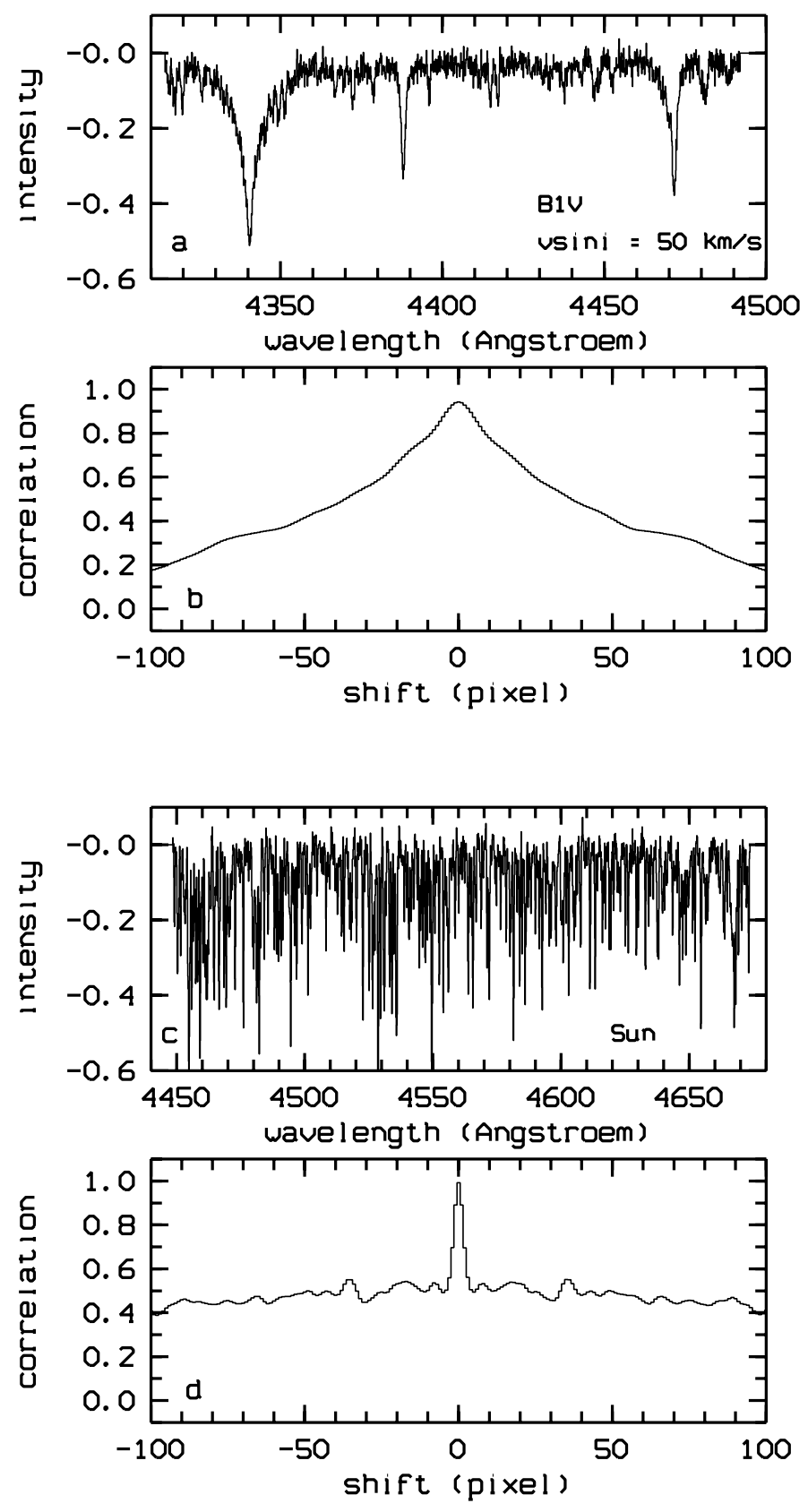

Fig. 1. a,b) Synthetic early-type spectrum with a continuum $S / N=50$, and the central part of its cross-correlation function with an intrinsically identical template of $S / N=200$. c,d) Idem for a solar spectrum

flux level. We use the dimensionless coordinate $p$ defined by the transformation $x=x_{0}+p \Delta x$ so that its integer values $1 . . N$ correspond to the central positions of the pixels and we conveniently refer to it as the "pixel number". Let $I(p)$ denote the sampled, normalised and noise-free spectrum, and $N_{\mathrm{O}}(p)$ and $N_{\mathrm{T}}(p)$ the random noise on object and template spectrum, respectively, at that same flux level. Following the argument of Brown (1989), we can calculate the apparent shift $\delta$, due to noise only, between 
these two spectra by minimizing

$$
f(\delta)=\sum_{p} W(p)\left[I(p)+N_{\mathrm{O}}(p)-I(p+\delta)-N_{\mathrm{T}}(p)\right]^{2} .
$$

Note that maximizing the cross-correlation function is indeed equivalent to a least-squares minimization of the difference between the two spectra (see e.g. Furenlid \& Furenlid 1990). The statistical weight $W(p)$ must equal the inverse of the variance on the difference spectrum:

$W(p)=\frac{1}{\left\langle N_{\mathrm{O}}^{2}(p)\right\rangle+\left\langle N_{\mathrm{T}}^{2}(p)\right\rangle}$

where \langle\rangle denotes the expectation value. Approximating $I(p+\delta) \cong I(p)+\delta \frac{\mathrm{d} I}{\mathrm{~d} p}$ for well sampled spectra and sufficiently small $\delta$, where the derivative is indeed the derivative of the sampling values with respect to the coordinate $p$, we obtain

$\delta=\frac{\sum_{p} W(p)\left[N_{\mathrm{O}}(p)-N_{\mathrm{T}}(p)\right] \frac{\mathrm{d} I}{\mathrm{~d} p}}{\sum_{p} W(p)\left(\frac{\mathrm{d} I}{\mathrm{~d} p}\right)^{2}}$.

Denoting the variance on $\delta$ by $\sigma^{2}=\left\langle\delta^{2}\right\rangle$, one finds

$$
\begin{aligned}
\sigma^{2} & =\frac{\sum_{p} W^{2}(p)\left(\frac{\mathrm{d} I}{\mathrm{~d} p}\right)^{2}\left(\left\langle N_{\mathrm{O}}^{2}(p)\right\rangle+\left\langle N_{\mathrm{T}}^{2}(p)\right\rangle\right)}{\left[\sum_{p} W(p)\left(\frac{\mathrm{d} I}{\mathrm{~d} p}\right)^{2}\right]^{2}} \\
& =\frac{1}{\sum_{p} \frac{1}{\left\langle N_{\mathrm{O}}^{2}(p)\right\rangle+\left\langle N_{\mathrm{T}}^{2}(p)\right\rangle}\left(\frac{\mathrm{d} I}{\mathrm{~d} p}\right)^{2}} \cdot
\end{aligned}
$$

For the sake of clarity, we repeat that $\left\langle N_{\mathrm{O}}^{2}(p)\right\rangle$ and $\left\langle N_{\mathrm{T}}^{2}(p)\right\rangle$ are the variances on both spectra after normalisation, and that $\sigma$ is expressed in units of pixel number $p$. If the spectra are binned into pixels of constant pixel-size in $x=$ $\log (\lambda)$, which is required for computing a Doppler shift, one must multiply $\sigma$ by the pixel-size in $\log (\lambda)$ units times $c$ to obtain a velocity. The standard deviation $\sigma$ represents a lower bound for the expected random error on the measured shift of one sampled and noisy spectrum with respect to an intrinsically identical one. In fact the derivation assumes that the underlying (noiseless) structure of the spectra is known with infinite resolution and that this information can be used to find the shift. Even though (4) will therefore not always provide a realistic error estimate, it is very important as a reference value.

A similar result has been obtained long before in signal-processing theory: if one replaces the statistical weight in (2) by a constant one describing additive, wavelength-independent noise, then the expression for the expected error on the time-delay of a radar signal is recovered exactly. This is particularly interesting since Woodward \& Davies (1950) derived this result without making a first-order approximation like the one leading to (3) so that this approximation probably does not restrict the validity of (4) to spectra with high $S / N$. However,
Woodward (1953) does point out that there is a $S / N$-level above which it certainly cannot be meaningful anymore, i.e. the so-called ambiguity limit which was estimated by Fellgett (1953) to be about 3 for astronomical spectra.

One can trivially rewrite the variances on object and template appearing in Eq. (4) in terms of their $S / N$ :

$$
\begin{aligned}
& \left\langle N_{\mathrm{O}}^{2}(p)\right\rangle+\left\langle N_{\mathrm{T}}^{2}(p)\right\rangle= \\
& \quad I^{2}(p)\left[\frac{1}{(S / N)_{\mathrm{O}}^{2}(p)}+\frac{1}{(S / N)_{\mathrm{T}}^{2}(p)}\right] .
\end{aligned}
$$

In the common case of spectra $I(p)$ being normalised to their continuum level, one may straightforwardly express the variances on these spectra as a function of their original continuum level $\left(C_{\mathrm{O}}(p), C_{\mathrm{T}}(p)\right)$ and of their original read-out noise $\left(R_{\mathrm{O}}, R_{\mathrm{T}}\right)$, both expressed in photon units:

$$
\begin{aligned}
& \left\langle N_{\mathrm{O}}^{2}(p)\right\rangle+\left\langle N_{\mathrm{T}}^{2}(p)\right\rangle= \\
& \quad I(p)\left(\frac{1}{C_{\mathrm{O}}(p)}+\frac{1}{C_{\mathrm{T}}(p)}\right)+\left(\frac{R_{\mathrm{O}}^{2}}{C_{\mathrm{O}}^{2}(p)}+\frac{R_{\mathrm{T}}^{2}}{C_{\mathrm{T}}^{2}(p)}\right) .
\end{aligned}
$$

Note that the continuum level and read-out noise in this expression refer to those of the one-dimensional spectrum, thus after a possible extraction.

In the case of pure photon noise, identical $S / N$ of object and template spectrum, a series of $L$ separated identical Gaussian absorption lines of width $\sigma_{1}$, central depth $d$ $(0<d \leq 1)$, and signal-to-noise in the continuum $(S / N)_{\mathrm{c}}$, Eq. (4) can be written as

$\sigma=\frac{\sqrt{2} \sqrt{\sigma_{1}}}{(S / N)_{\mathrm{c}} \sqrt{L} d \mathrm{f}(d)}$

where the only approximation made is replacing the sum by an integral. $\mathrm{f}(d)$ is a weak monotonous function of $d$, being 0.94 for $d \rightarrow 0$ and 2.01 for $d=1$. $\sigma$ and $\sigma_{1}$ are expressed in pixels. The factor $\sqrt{2}$ is absent in case of a noise-free template.

For Gaussian emission lines with a peak signal-to-noise in their centre equal to $(S / N)_{\mathrm{p}}$, a negligible continuum intensity, and otherwise similar parameters as above, one finds

$\sigma=\frac{\sqrt{2} \sqrt{\sigma_{1}}}{(S / N)_{\mathrm{p}} \sqrt{L}}(2 \pi)^{-0.25}$

where the last numerical factor is about 0.63. Both the latter expressions may be useful for quick estimates of the intrinsic radial velocity precision of a (portion of a) spectrum, and give insight in the dependence of that precision on the spectrum parameters.

\section{Cross-correlation peak centering}

\subsection{General remarks}

We assume now we have obtained the cross-correlation function of an object spectrum and an intrinsically 
(i.e. apart from the noise) identical template spectrum and we are faced with the problem of finding its maximum position with the best possible precision. Since the width of even the narrowest stellar absorption lines is usually much larger than the positional error one is prepared to tolerate, spectra intended for radial-velocity measurements must be well oversampled. The actual degree of oversampling for the resulting cross-correlation function may vary widely, from typically 5 or 6 pixels within the central peak for late-type spectra to sometimes well over 100 for early-types. Even so, in most cases the sampling step is still larger than the tolerable error and one needs some form of interpolation between the sampling values of the correlation peak to obtain the required (sub-pixel) precision.

In practice one cannot expect automatically to obtain a precision corresponding to the lower bound for the random error discussed in the previous section. Strictly speaking, this would require an exact model with infinite resolution for the correlation peak. Nevertheless, if the spectra contain nothing but well-separated unbroadened metal lines then a Gaussian model for the cross-correlation function may come close to this ideal situation. Most often, however, line blending and the mixture of $\mathrm{H}, \mathrm{He}$ and rotationally broadened metal lines in early-type stars give the correlation peak a complicated structure for which no well-matching analytical model exists (see Fig. 1) and then, depending on the technique used to interpolate between the sampling values of the correlation peak, the expected random error at the sub-pixel level may become much larger than the lower bound. Tonry \& Davis (1979) already used a combination of two kinds of low-pass filtering (general purpose Fourier filtering and the fit of a parabola to the full width of the correlation peak) for the reduction of noise effects in the case of late-type spectra. Below we argue that this is in fact essential in the case of early types and we study how such a combination should be tuned.

Qualitatively one can argue as follows. The substructure of the correlation peak, away from the centre, is due to the overlap of non-corresponding features in the spectra so it is irrelevant for the localization of the correlation maximum. If only low-pass Fourier filtering is applied and the correlation maximum is obtained correspondingly by Fourier-interpolation of the filtered peak, then that substructure will inevitably influence the position. On the other hand, if only a model-fit were applied, then again the substructure would influence the position of the maximum unless the model were a perfect match. So we need to eliminate the influence of the spurious substructure as far as possible. Obviously this can be done by fitting a model only to the very central part of the correlation peak. But then only few samples are used and the noise on these could strongly increase the random error. Hence the need for low-pass filtering prior to the model fit. The combined procedure of course requires two "smoothing parameters" to be chosen (fit-interval and Fourier-bandwidth); we must also address the question of which model to choose for the fit.

\subsection{Fit-points and fit-functions}

In order to investigate how the random error behaves as a function of the number of fit-points, and when different polynomial models for the correlation peak are used, Monte-Carlo experiments were conducted as follows: an object and a template spectrum are created by twice adding photon and read-out noise with a given total $\mathrm{rms}$ in the continuum (corresponding to a given continuum $S / N$ ) to a synthetic spectrum, which may have been rotationally broadened. In all our experiments, we fix the continuum $S / N$ for the template spectrum at the fairly high value of 200 , and fix the read-out noise for both object and template at $10 \mathrm{e}^{-}$. For details on the synthetic spectra we refer to Vrancken et al. (1997). All spectra have a 1 pixel resolution of $2810^{-6}$ in $\ln (\lambda)( \pm 0.13 \AA / p$ at $4500 \AA)$ but as long as the spectra are well sampled, all our conclusions are independent of resolution.

The spectra are then cross-correlated using the CORSPEC package (updated from Verschueren 1991) and the maximum position of the cross-correlation peak is fitted using a number of fit-points ranging from 5 to twice its $F W H M$ in steps of 2 pixels, and using 3 different fit-functions: a $2^{\text {nd }}$ degree, a symmetric $4^{\text {th }}$ degree, and an ordinary 4th degree polynomial. Obviously, with our experimental set-up, a symmetric fit-function is appropriate. In the case of observed spectra, however, small systematic mismatch between object and template may give reason to use a fit-function allowing for asymmetry. Therefore, the ordinary $4^{\text {th }}$ degree polynomial is included. Note that a $3^{\text {rd }}$ degree polynomial is unsuitable because its uneven highest order term causes unacceptably high sensitivity for asymmetries in the random noise. The use of a Gaussian fit-function is also less appropriate since its parameters are not well constrained by information from the mere top of a peaked function (unless one constrains the background term, but this value is not known a priori for early-type spectra). Discretisation errors during centering are minimized according to the prescription given in David \& Verschueren (1995). This process is repeated $n=2000$ times with independent random noise, and the rms of the fitted central positions, representing the random error on a single measurement of the shift, is computed for each value of the number of fit-points and for each fit-function. The relative error on such a rms is $\sim 1 / \sqrt{2 n}=0.016$.

Figure 2 shows an example and compares with the lower bound for the random error discussed in Sect. 2. The details of the curves depend on the spectral characteristics and on the $S / N$ of object and template, but the following important properties are typical: 


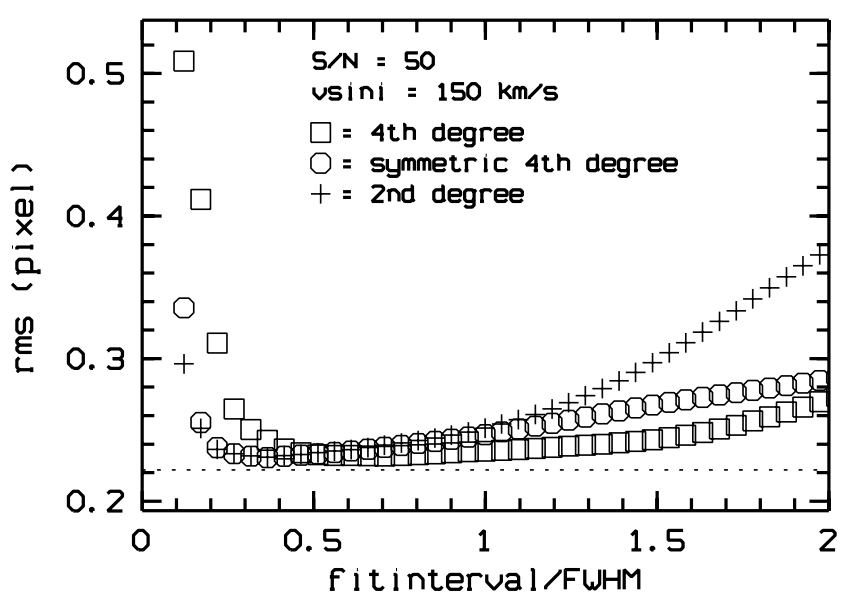

Fig. 2. Random error on a cross-correlation shift as a function of the length of the fit-interval used, and for the three polynomial fit-functions indicated; it is derived from a Monte-Carlo experiment with 2000 runs. The lengths of the fit-interval are normalised to the $F W H M$ of the cross-correlation peak (about 40 pixels in this case); they correspond to a number of fit-points starting from 5 pixels with increments of 2 . A B1V synthetic spectrum in the region $4382-4759 \AA$ was used with the indicated rotational velocity and object continuum $S / N$. The dashed line indicates the level of the lower bound from Sect. 2

- There exists an optimal number of fit-points $n_{\mathrm{opt}}$ around the cross-correlation peak maximum for which the random error is statistically smallest; it is only very weakly dependent on the fit-function.

- The actual minimum random error agrees very well with the theoretical lower bound. The maximum discrepancy between both occurs for broad-lined stars with a low $S / N$ for which the Monte-Carlo minimum is about $5 \%$ higher than the lower bound.

- For a number of fit-points smaller than $n_{\text {opt }}$, overfitting increases the random error, and more so the more free parameters the fit-function contains. This rise in random error is more pronounced with a broader cross-correlation peak and if more (noisy) continuum pixels are included in the spectrum. For a number of fit-points above the optimal one, the errors are becoming more and more dominated by mismatch between the fit-function and the cross-correlation peak.

In order to minimize the random error, one would thus like to fit the cross-correlation function using the optimal number of fit-points for all spectra. However, in practice the value of $n_{\mathrm{opt}}$ is not known a priori since it depends intricately on the spectral characteristics. Moreover, it is preferable to use as few fit-points as possible for reasons mentioned in Sect. 3.1. Therefore, we investigated whether high-frequency Fourier filtering could provide a solution for both constraints.

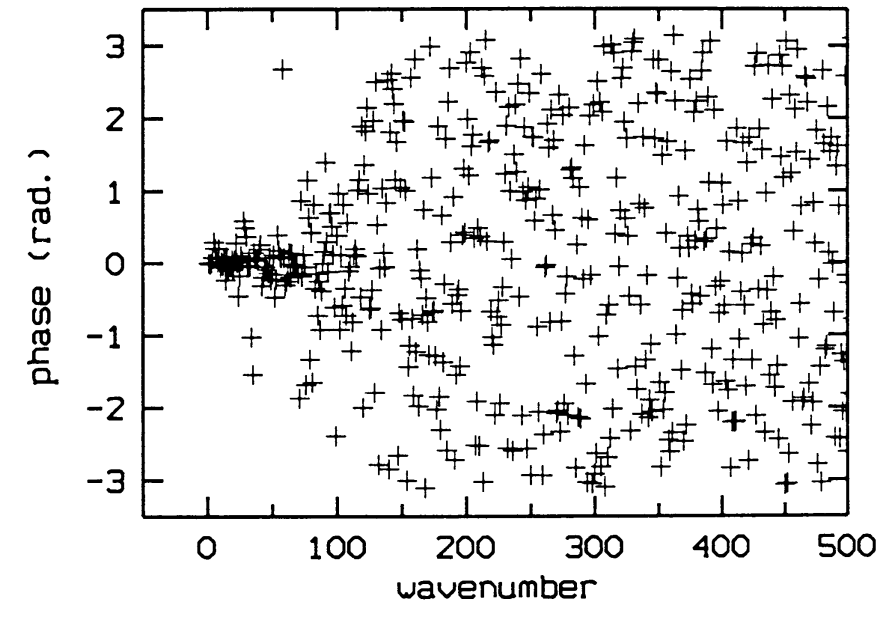

Fig. 3. Phase of the Fourier transform of the cross-correlation function versus wavenumber for an arbitrary couple of spectra used for Fig. 2. The $x$-axis is truncated for clarity

\subsection{High-frequency filtering}

The optimal cut-off frequency (or wavenumber) $f_{\mathrm{c}}$ in Fourier space may be derived from a plot of the phase of the Fourier transform of the cross-correlation function versus its frequency. Figure 3 shows, for 2 arbitrary spectra from the set used for Fig. 2, a linear behaviour for frequencies dominated by intrinsic spectral information, a region where the phase is randomized for noise-dominated frequencies, and an intermediate region. $f_{\mathrm{c}} \cong 250$ seems a safe choice for removing only fully noise-dominated information in this case. $f_{\mathrm{c}}$ can conveniently be expressed as $N / i$ with $N$ the total number of pixels in the spectrum. The "scaled filter value" $i=N / f_{\mathrm{c}}$ defined in this way is independent of the exact length of the spectrum; it is also independent of the sampling rate (i.e. the spectral resolution) provided that the spectra are well sampled and that the $S / N$ per pixel remains the same when changing the resolution. The optimal filter $i$ thus only depends on the type of spectrum (i.e. intrinsic characteristics of the lines and rotational velocity) and on its $S / N$. For the spectrum used in Figs. 2 and 3 (for which $N=4096$ ), $f_{\mathrm{c}} \cong 250$ thus corresponds to $N / 16$ filtering $(i=16)$. Note that one can always choose $i \geq 4$, with $N / 4$ filtering being optimal for spectral lines with a $F W H M$ equal to the 2 pixel spectral resolution. Since our filter choice is always very conservative, i.e. only fully noise-dominated frequencies are removed, a simple rectangular filter can be used.

Figure 4 then shows the influence of successively increased filtering on the random error from an identical Monte-Carlo experiment as in Fig. 2, and for the quadratic fit-function. The behaviour is again typical for all spectra: filtering up to the optimal cut-off frequency does not significantly change the minimum random error, but allows to attain it in practice for all fit-intervals smaller than the optimal one. This allows one to fit cross-correlation peaks close to their centre with a fixed number of fit-points, and 


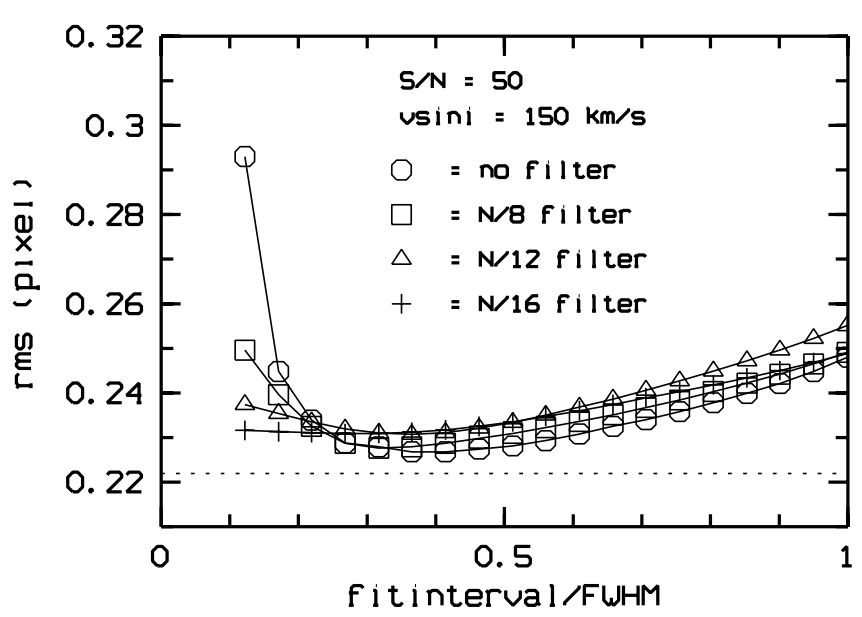

Fig. 4. Idem as Fig. 2, but now showing also the effect of 3 different high-frequency filters. The results are shown only for the quadratic fit-function. The small rise in minimum rms for the last two filters is insignificant and is an occasional effect that does not necessarily occur for other spectral regions or parameters

ensures an optimal precision at the same time. Further tests have shown that filtering significantly beyond the cut-off frequency increases the minimum random error accordingly due to a degradation of the intrinsic spectral features. Note that, although high-frequency filtering apparently increases the $S / N$ of the spectra, it can not improve the actual optimal precision attainable from those spectra: indeed, the latter is obviously limited by those random-noise frequencies that are also present in the intrinsic spectral information itself.

Since it is unpractical and often ambiguous to determine $f_{\mathrm{c}}$ or $i$ from plots such as Fig. 3 for each given pair of spectra, we investigated its dependence on spectral characteristics and on $S / N$. This was done in a set of Monte-Carlo experiments (1000 runs) with different synthetic spectra (only metal lines in a B1V spectrum, only $\mathrm{H}$ lines in a B8V spectrum, and each for $v \sin i=5,50,100$, 150,200 and $300 \mathrm{~km} \mathrm{~s}^{-1}$ ) and with different values of the $S / N$ (50 and 200). In each case, the Monte-Carlo rms of the phase of the Fourier transform of the cross-correlation function was computed as a function of Fourier frequency $f$, and compared to the value of $1.81 \mathrm{rad}$ which corresponds to a random phase between $-\pi$ and $+\pi$.

Figure 5a shows an example of such an experiment with the same input parameters as used in Fig. 2; the plot is already made as a function of $N / f=i$ which allows direct reading of the scaled filter value to be used. For high values of $i$ (small frequencies), spectral information still dominates and produces low rms values (note also the smaller rms around frequencies related to the rotational broadening function, see Gray 1976). A rms of about $1.8 \mathrm{rad}$ is reached below a cut-off value of $i \cong 15$ which corresponds well to the value of 16 derived earlier from

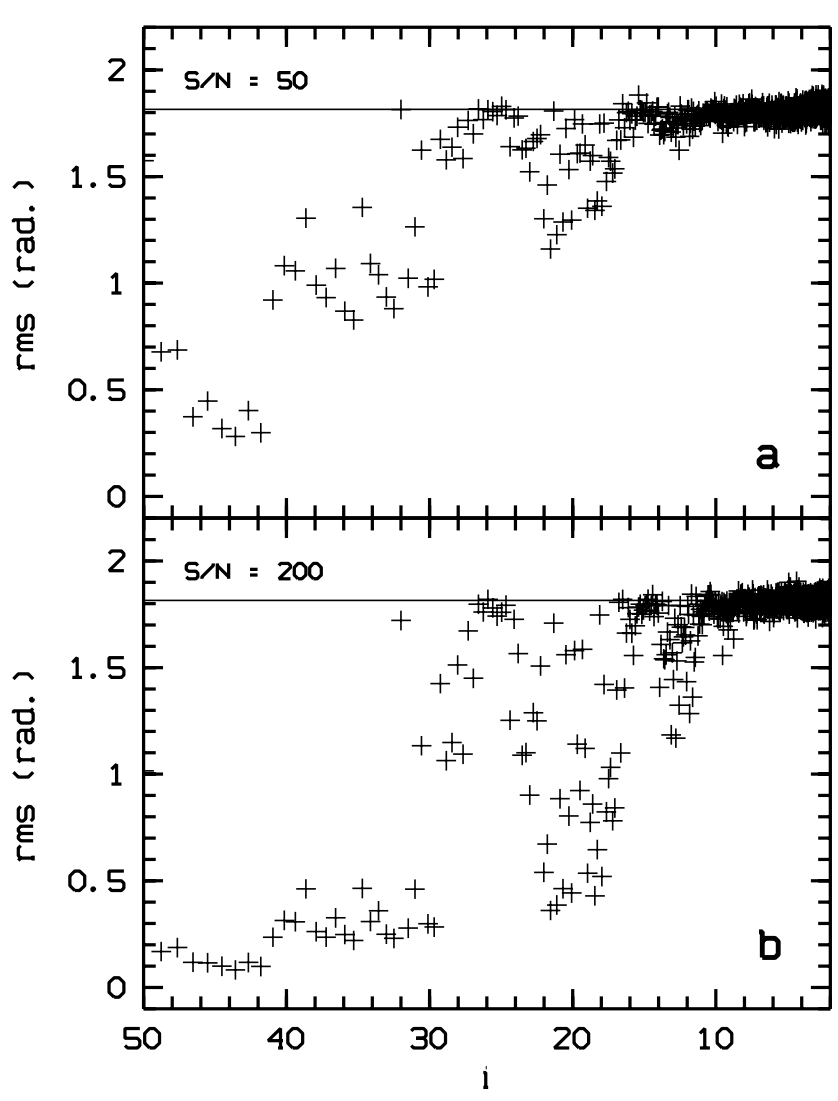

Fig. 5. a) Monte-Carlo rms of the phase of the Fourier transform of the cross-correlation function versus scaled filter value $i=N / f$. The same input parameters were used as for Fig. 2 . The $x$-axis is truncated towards higher values for clarity. The range $i=50$ to 2 corresponds to wavenumber $=82$ to 2048 in Fig. 3. The full line corresponds to full randomization of the phase. b) Idem but now for a $S / N=200$

a single spectrum (Fig. 3) and to the value empirically needed to flatten the curve in Fig. 4.

In order to investigate the influence of the spectrum $S / N$, the same Monte-Carlo experiment was repeated with a $S / N=200$. Figure $5 \mathrm{~b}$ shows that a cut-off value of $i \cong$ 10 seems more appropriate now due to the fact that intrinsic spectral information now beats the noise up to higher frequencies. From the whole set of experiments performed on other spectra, we can indeed generally conclude that a more pronounced filtering may be applied in case of lower $S / N$.

The influence of rotational velocity (directly influencing line-width) was examined by performing all Monte-Carlo experiments for the 6 values of $v \sin i$ mentioned above. Figure 6 summarizes the results for the two different values of the $S / N$ and for metal and $\mathrm{H}$-lines separately. For a given spectrum sampled in $N$ pixels, the adequate value of $i$ may be read from these curves and can be used to compute the optimal cut-off frequency $f_{\mathrm{c}}=N / i$ for high-frequency filtering, independent of 


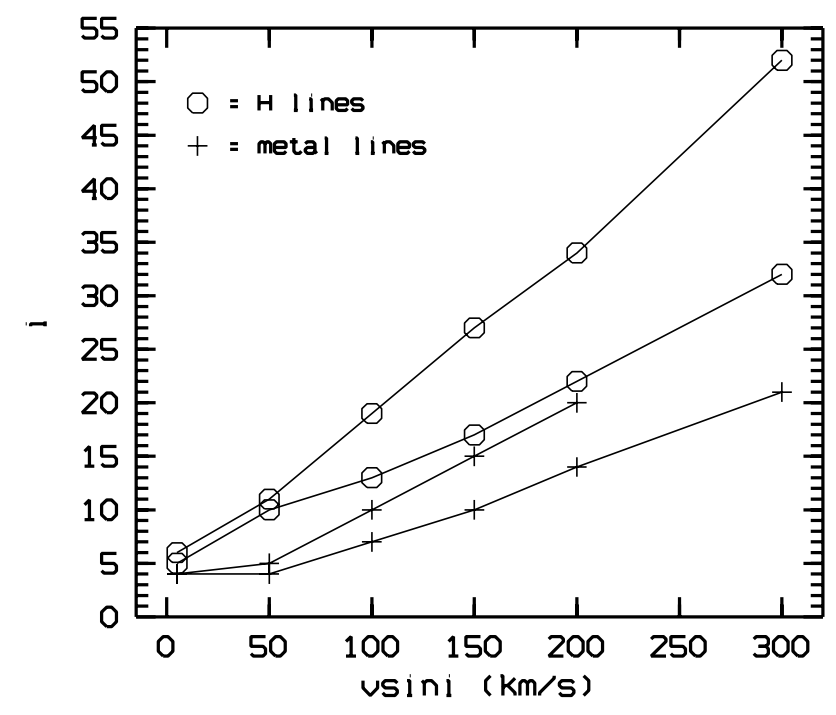

Fig. 6. Optimal scaled filter value $i=N / f_{\mathrm{c}}$ for high-frequency filtering as a function of rotational velocity, for spectra containing metal respectively $\mathrm{H}$ lines as indicated. In each case, the lower value refers to an object spectrum $S / N=200$ and the higher one to a $S / N=50$

spectral resolution (for well resolved spectra).

Finally, we verified that the conclusions found in this section are valid independently of the fit-function used. However, we give preference to keeping the number of free parameters as small as possible, especially since this yields the smallest increase in random error in case the chosen degree of filtering was not optimal (see Fig. 2). In addition, for spectra without systematic mismatch, the fit-function needs to be symmetric. Our proposed fitting method is therefore a parabolic fit to the highest 5 pixels of the cross-correlation function, after applying a degree of high-frequency filtering determined from Fig. 6 .

\section{Rotational object-template mismatch}

The question we raise here is how the random error on a cross-correlation derived radial velocity shift behaves when the template has a lower rotational velocity than the, otherwise intrinsically identical, object spectrum.

Monte-Carlo tests were performed similar to the ones described in Sect. 3: a sharp-lined synthetic spectrum was once broadened to a given value of $v \sin i$ to yield the object spectrum, and once broadened to several lower $v \sin i$ values to simulate different template spectra. Random noise was independently added to both and the shift was computed after cross-correlation. No Fourier filtering was applied in order to show the pure effect of rotational mismatch. As an example, Fig. 7a shows the rms of these shifts for different values of the rotational velocity of the template. The conclusion is that rotational mismatch does

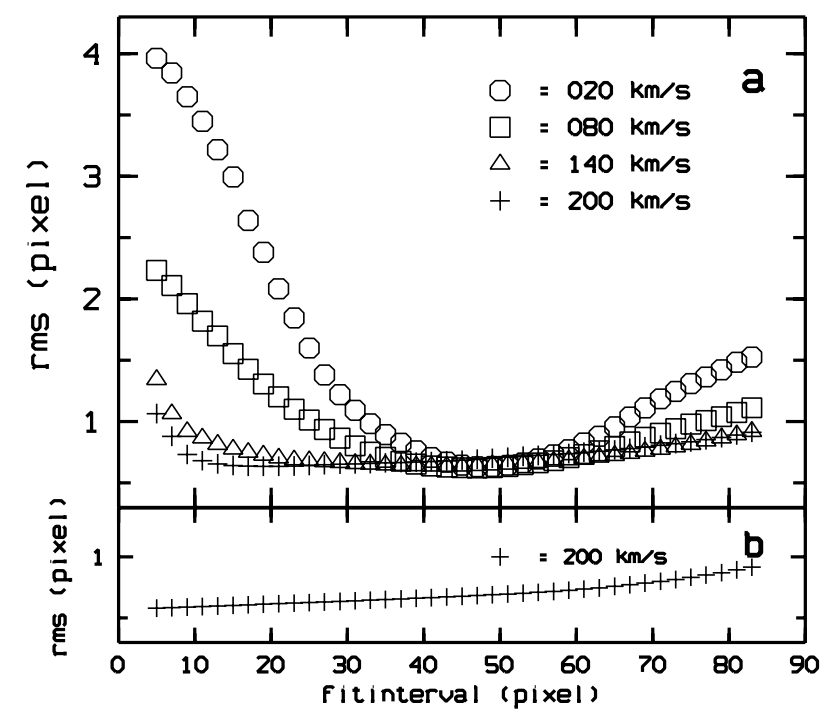

Fig. 7. a) Random error on a cross-correlation shift as a function of the number of fit-points used (for the quadratic fitfunction) as derived from a Monte-Carlo experiment with 1000 runs. A B1V synthetic spectrum in the region $4547-4759 \AA$ was used. The object spectrum has a rotational velocity of $200 \mathrm{~km} \mathrm{~s}^{-1}$ and a $S / N=50$. The rotational velocity of the template spectrum varies as indicated and its $S / N$ was 200 . The lower bound for the random error (Sect. 2), in absence of rotational mismatch, is 0.58 pixel. Note that the fit-interval is not normalised here to the $F W H M$ of the cross-correlation peak since the latter changes with rotational mismatch (from 35 to 39 pixels). b) Idem as a) but now the rotational broadening of the template to $200 \mathrm{~km} \mathrm{~s}^{-1}$ was done after the noise was added

not increase the minimum random error obtainable in case the spectra are rotationally matching; this minimum error, however, can now be achieved in practice only for much larger fit-intervals, having lengths within a smaller range (in this case between $\sim 45$ and 55 pixels). The reason is that the random noise on the cross-correlation function has increased due to the poorer line overlap.

The last curve of the experiment just described correponds to the case of using an observed template with a $v \sin i$ matching the object as close as possible. Alternatively, one may also use a template consisting of an artificially rotationally broadened version of a sharp-lined observed spectrum. The important difference between the two cases is that in the latter, rotational broadening is done after addition of noise. Figure $7 \mathrm{~b}$ shows the effect on the random error in the latter case: the minimum random error obtainable remains roughly unaltered, but it can now be achieved for the smallest number of fit-points possible. This result is, not surprisingly, very similar to the effect of high-frequency filtering described in Sect. 3.3 since rotational broadening obviously smooths the spectrum; clearly, high-frequency 
filtering of the spectra would turn the last curve of Fig. 7a into that of Fig. 7b.

We conclude that rotational mismatch should be avoided for technical reasons only, namely for its implication on the fitting process of the cross-correlation peak. The decision to use either observed rotationally matching templates in combination with high-frequency filtering, or artificially broadened sharp-lined templates, must be based on the following two factors: on the one hand the availability of an observed rotationally matching template spectrum, and on the other hand the adequacy of straightforward rotational broadening to mimic $v \sin i$ (which becomes questionable for $v$ close to the break-up velocity; see e.g. Collins \& Truax 1995).

\section{Actual random error: The $r$-statistic revisited}

In the previous two sections we discussed a technique to make the random error approach its lower bound quite closely but, whatever procedure one follows, one will need to assess the actual random error on a radial velocity measurement. In order to do so one can always apply straightforward error progression which naturally takes into account the effect of all operations actually performed on the data. The result for a simple case is given in the Appendix (Eq. (A7)), but one immediately perceives that its evaluation is rather tedious. This may be the reason why alternatives have been sought such as error progression with strong approximations (Murdoch \& Hearnshaw 1991), the lower bound (Sect. 2) itself, or the so-called $r$-statistic.

Tonry \& Davis (1979) computed the well known error estimate $\epsilon(r)$ on a cross-correlation derived radial velocity shift, using only properties of the cross-correlation function itself:

$$
\begin{aligned}
\epsilon & =\frac{k}{1+r} \\
r & =\frac{h}{\sqrt{2} \sigma_{\mathrm{a}}} \\
\sigma_{\mathrm{a}}{ }^{2} & =\frac{1}{2 N} \sum_{n}[c(\delta+n)-c(\delta-n)]^{2}
\end{aligned}
$$

where $h$ is the peak height of the normalised crosscorrelation function $c(n), \sigma_{\mathrm{a}}$ the rms of its antisymmetric component, and $k$ a proportionality constant. $\epsilon$ is expressed in pixels. Using statistical arguments, they concluded that this constant $k$ must equal about $N / 8 B$ with $B$ the wavenumber for which the Fourier transform of the cross-correlation function reaches half maximum. They found good agreement with independently known errors in the case of galaxy spectra.

Peterson (1983) reported differences, which correlated with the line width of the spectrum, between this theoretical error estimate and the true errors. Experimenting with simple synthetic spectra and (portions of) early-type spectra, Verschueren (1991) found that estimating $B$ in practice was to a large extent arbitrary. He found empirically that $k \cong 0.5 w$, with $w$ the $F W H M$ of the crosscorrelation peak, gives excellent agreement with MonteCarlo simulated random errors. Kurtz et al. (1992) then showed analytically that, for sinusoidal noise with a halfwidth equal to the half-width of the correlation peak, $k$ must equal $3 w / 8$, which is now widely in use.

The $r$-parameter was intended to describe systematic object-template mismatch as well as random noise (Tonry, private communication, 1998) and was developed and successfully tested on late-type spectra (see Kurtz \& Mink 1998 for an update and overview). In this section, we investigate the applicability of the so-called $r$-statistic to purely random errors (i.e. in the absence of systematic mismatch) for early-type spectra.

Caution when applying the $r$-statistic to other than late-type spectra is justified since, in a strict sense, the relation (9) does not exist. For the simple case described in the Appendix, the explicit expressions (A7), (A10) show that the expected squared error $\sigma_{\mathrm{P}}{ }^{2}$ and the expected $\left\langle\sigma_{\mathrm{a}}{ }^{2}\right\rangle$ depend on the structure of the spectrum in a different way: both take the form of a weighted average of the flux variances on individual pixels, but the weights (A8), (A11) may be quite different; moreover, $\sigma_{\mathrm{P}}{ }^{2}$ depends on the fit-interval while $\left\langle\sigma_{\mathrm{a}}{ }^{2}\right\rangle$ does not. So, in general one cannot expect to find a relation between the two which is independent of the nature of the spectrum. While Monte-Carlo simulations with a given spectrum and different noise levels will reveal a strict correlation between the observed error and $\sigma_{\mathrm{a}}$, the actual relation between those quantities must depend on the structure of the spectrum. In other words, there cannot be a "generally valid" expression for $k$. The fact that the error estimate (9) does appear to work for all late-type spectra must be due to the fact that the latter, viewed over a sufficiently large wavelength region and with a pixel-size not much smaller than the typical width of a spectral feature, all do have the appearance of a strongly fluctuating function: as a consequence, the weights (A8) and (A11) become approximately independent of $n$. In fact, this appearance of late-type stars corresponds qualitatively to the main condition imposed by Tonry \& Davis (1979), that the Fourier power spectrum of the "perfect correlation function" (i.e. the signal function, $c_{0}(i)$ in our notation) should be very similar to the one of the "remainder function" (i.e. the noise function $\left.c(i)-c_{0}(i)\right)$. Since the expression (9) has the benefit of being simple, elegant and intuitively appealing, we have investigated in detail whether it might also in some way be applied to early-type spectra.

For early-type spectra, which generally contain broader lines, and/or when cross-correlating selected small spectral regions (as often required for such spectra, see e.g. the arguments in Verschueren 1995; Verschueren et al. 1999b), the cross-correlation function consists of much broader features than is the case for late-type stars. In order to illustrate this point in a very simple way, we 
Table 1. Monte-Carlo computation (2000 runs) of the expected random error on a radial velocity shift. The spectrum was a single Gaussian absorption line of $F W H M=16$ pixels and a central depth of $40 \%$. Photon and read-out noise were added to both object and template, consistent with a continuum $S / N$ of 100 and 200, respectively. The corresponding true radial velocity precision is 0.068 pixel (from the Monte-Carlo dispersion, or from Eq. (4)). Average results are given as a function of the total number of pixels $N$ in the spectrum. $\left\langle\sigma_{\mathrm{a}}\right\rangle$ is computed with respect to the theoretical centre of the cross-correlation function (0 pixel), see text

\begin{tabular}{rcccc}
\hline$N$ & $\begin{array}{c}w \\
\text { (pixel) }\end{array}$ & $\langle h\rangle$ & $\left\langle\sigma_{\mathrm{a}}\right\rangle$ & $\begin{array}{c}\langle\epsilon\rangle \\
\text { (pixel) }\end{array}$ \\
\hline 32 & 22.6 & 1.00 & 0.0037 & 0.044 \\
64 & 22.6 & 1.00 & 0.0050 & 0.059 \\
128 & 22.6 & 1.00 & 0.0053 & 0.063 \\
256 & 22.6 & 0.99 & 0.0055 & 0.066 \\
512 & 22.6 & 0.98 & 0.0055 & 0.066 \\
\hline
\end{tabular}

performed a Monte-Carlo experiment using a spectrum consisting of one Gaussian absorption line surrounded by different amounts of continuum. Table 1 shows that only when the cross-correlation function predominantly consists of relatively narrow features (large $N$ ), the correct error is obtained from $\epsilon$; for small $N$, the cross-correlation function is dominated by the relatively broad central peak, resulting in a too low value for the error owing to a decreased $\sigma_{\mathrm{a}}$.

Similar experiments were then performed on different spectral regions of synthetic early-type spectra, comparing the error estimate $\epsilon$ with the true random error from the Monte-Carlo dispersion for different amounts of added random noise. Figure 8 shows results for 4 different spectra. These results are only indicative for the differences between $\epsilon$ and the true error that may occur, and should not be used in a quantitative sense. In general, Gaussian line shapes or rotation profiles yield too small error estimates $\epsilon$, while lorentzian line shapes produce too large values probably due to their relatively large $F W H M w$. We conclude that, except for late-type spectra, the predicted error $\epsilon$ may be very incorrect, and that moreover a generalisation to early-type spectra would be impossible because the required proportionality constant $k$ would depend on the details of each individually selected spectrum (e.g. line shape, amount of continuum pixels included as shown in Table 1, mixture of metallic, $\mathrm{He}$ and $\mathrm{H}$ lines, etc.).

Two other important problems related to the practical computation of $\epsilon$ in the framework of early-type stars are worth mentioning. First, the cross-correlation function is often composed of overlapping broad components so that the $F W H M w$ of the central peak looses the significance assumed in the $r$-statistic. Secondly, it is assumed (Tonry $\&$ Davis 1979) that $\sigma_{\mathrm{a}}$ is computed with respect to the exact central position of the cross-correlation function. In

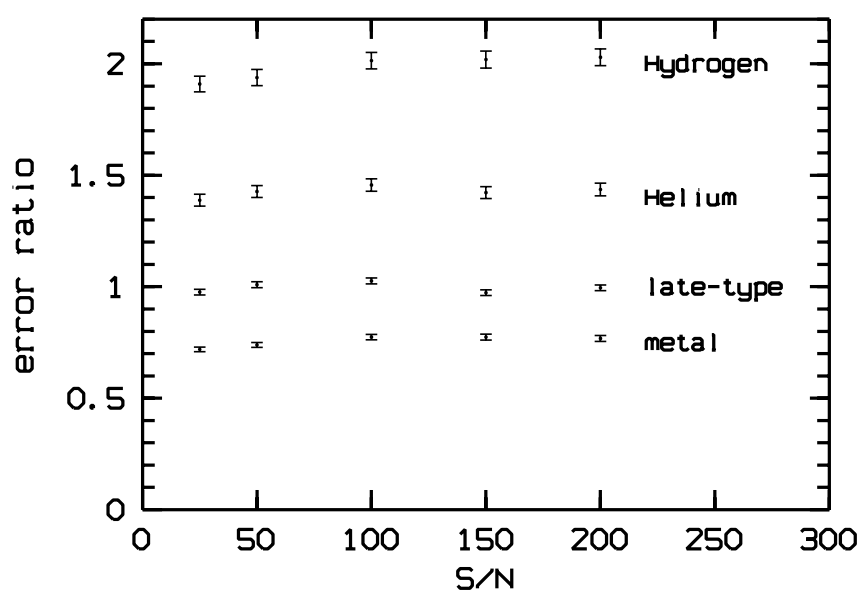

Fig. 8. Ratio of the error $\epsilon$, predicted by the $r$-statistic and computed as the mean from a Monte-Carlo experiment, to the true random error from the Monte-Carlo dispersion. Results are given as a function of object spectrum $S / N$ (the $S / N$ of the template was fixed at 200) for 4 different spectra: a late-type (solar) spectrum $(4448-4671 \AA$ ), a metal line region in a synthetic B1V spectrum with $v \sin i=150 \mathrm{~km} \mathrm{~s}^{-1}(4547-4759 \AA)$, a He line in a synthetic B1V spectrum with $v \sin i=50 \mathrm{~km} \mathrm{~s}^{-1}$ $(4020-4032 \AA)$ and a $\mathrm{H}$ line in a synthetic B8V spectrum with $v \sin i=50 \mathrm{~km} \mathrm{~s}^{-1}(4281-4381 \AA) .\left\langle\sigma_{\mathrm{a}}\right\rangle$ was computed with respect to the theoretical centre of the cross-correlation function (0 pixel), see text. Error bars take into account the statistical error on both computed error estimates

practice, one evidently has to use the measured central position, which yields the same result provided that the width of the central peak is much smaller than the complete cross-correlation function. In case small spectral regions are selected, the computation of $\sigma_{\mathrm{a}}$ with respect to the measured centre can be quite different from its correct value. For example, the value of $\langle\epsilon\rangle$ in Table 1 for $N=32$ and $N=64$ would (further) decrease to 0.009 and 0.045 , respectively, if computed with respect to the measured centre of the cross-correlation peak.

\section{Conclusions}

In order to approach optimal radial velocity precision for early-type stars, a number of precautions have to be taken into account to reduce random errors to levels which are attained effortlessly for late-type spectra.

We generalised the theoretical lower bound for the random error attainable as a function of the intrinsic spectrum morphology and of the wavelength-dependent noise on object and template spectrum (Eq. (4)). It is valid for all (late and early-type) well sampled spectra. It shows that, for other than small rotational velocities, a high $S / N$ and a large wavelength region are required to beat the random errors sufficiently in early-type spectra (Eqs. $(7,8))$. 
Owing to the complex morphology of a crosscorrelation peak derived from blended and broadened early-type spectra, containing mixtures of metal, He and $\mathrm{H}$ lines, specific centering techniques are required to approach this lower bound for the random error in practice (even in the absence of systematic mismatch). Based on a wide range of Monte-Carlo experiments with synthetic early-type spectra, an optimal cross-correlation peak centering method is proposed: merely the top of the peak ( 5 pixels) is fitted with a low-order polynomial $\left(2^{\text {nd }}\right.$ degree $)$, after a well chosen high-frequency Fourier filtering has been applied. The filtering allows to approach very closely the lower bound for the random error using a very small amount of fit-points around the maximum position of the cross-correlation peak; using merely the centre of the cross-correlation peak is desirable because of the fact that there the radial velocity information is least perturbed, and for practical reasons. The optimal filter is computed as a function of the rotational velocity of the spectrum, while it also varies, to a lesser degree, with $S / N$ (Fig. 6).

Monte-Carlo experiments with synthetic spectra show that rotational mismatch, i.e. cross-correlating a rotationally broadened object with a narrow-lined template, does not increase (nor decrease) the minimum random error attainable with a broad-lined template. It is, however, virtually impossible to attain this minimum error in practice since it is connected to a very small range of fit-intervals of an a priori unknown length (Fig. 7). Using a template of roughly equal rotational velocity (and preferably equal inclination angle) as the object spectrum, in combination with high-frequency filtering, is therefore mandatory. Note that $v \sin i$ matching was also recommended by Peterson et al. (1984) in order to avoid multiple components of (nearly) equal strength on the cross-correlation peak in spectral regions with blended lines.

Finally, we investigated whether the error estimate based on the $r$-statistic (Tonry \& Davis 1979; Kurtz \& Mink 1998) can be used to predict the actual random error on a cross-correlation derived radial velocity shift between early-type stars. We showed that the actual relation between the true error $\epsilon$ and the antisymmetric part of the cross-correlation function $\sigma_{\mathrm{a}}$, theoretically depends on the structure of the spectrum (Appendix). Owing to their strongly fluctuating nature and their similar appearance, one could nevertheless find a "generally valid" relation between $\epsilon$ and $\sigma_{\text {a }}$ for late-type spectra. Our Monte-Carlo experiments with different types of earlytype spectra, however, show that this is impossible for the latter (Fig. 8). We conclude that its use is inadequate for early-type spectra for both theoretical and practical reasons.

Acknowledgements. We thank Herman Hensberge for many stimulating discussions, Myriam Vrancken for kindly providing the synthetic spectra, and the referee for valuable comments. WV acknowledges substantial financial support from the Fund for Scientific Research - Flanders (Belgium) (F.W.O.) through Research Grant No. 1.5.549.98.

\section{Appendix A: Properties of the cross-correlation function}

\section{Centering}

Let $c(i)$ be a discretized function with a prominent peak, the position of which is to be determined. As in Tonry \& Davis (1979) we do so by finding the position of the maximum of a parabola fitted to the peak. Let $i_{\mathrm{c}}$ denote the pixel with the highest value; if one uses an odd number of pixels (say, $2 p+1$ ) chosen symmetrically around $i_{\mathrm{c}}$, then the position of the maximum (expressed in pixels) can be written as $i_{\mathrm{c}}+\delta$ and the pixel fraction $\delta$ is given by:

$\delta=K m_{1}, \quad K=\frac{4 p^{2}+4 p-3}{5 p(p+1) m_{0}-15 m_{2}}$

where $m_{l}$ denotes the $l^{\text {th }}$ moment of the cross-correlation peak (within the interval used in the fit):

$m_{l}=\sum_{j=-p}^{p} j^{l} c(j)$

With an even number of pixels chosen symmetrically around the highest two values, a similar expression can be obtained; if necessary both results can be combined to reduce the discretization error as discussed in David \& Verschueren (1995).

\section{Random errors}

The random error on the position of a cross-correlation peak, i.e. the part of the error which is due to noise on the spectra, can be estimated in a straightforward way as the square root of the variance of the shift measured between two spectra, say $S$ and $T$, which are intrinsically identical and unshifted with respect to one another. We assume the spectra to be binned on $N$ pixels so that

$$
S(n)=S_{0}(n)+N_{\mathrm{S}}(n), \quad T(n)=S_{0}(n)+N_{\mathrm{T}}(n),
$$

where the subscript 0 indicates the intrinsic part and $N(n)$ represents the noise. We shall assume throughout that the noise on any pixel is statistically independent from the noise on all others. This assumption may not always be valid in practice, e.g. if the data have been rebinned at some stage of the data-reduction process or if they have been filtered prior to cross-correlation; in that case the results derived below will have to be modified but the argument remains essentially the same. Let us define

$$
n \oplus i \equiv n+i+N \quad(\bmod N)
$$$$
n \ominus i \equiv n-i+N \quad(\bmod N) \text {. }
$$ 
Then the correlation function can be written as:

$$
\begin{aligned}
c(i) & =\frac{1}{C} \sum_{n=0}^{N-1} S(n) T(n \oplus i), i=-N / 2, \ldots, N / 2-1 \\
C & =\sum_{n=0}^{N-1} S_{0}^{2}(n)
\end{aligned}
$$

where we have assumed, conveniently but without loss of generality, that $N$ is even. Similarly, we limit ourselves to the case where we can fit an odd number of pixels and we can apply Eq. (A1) (with $i_{\mathrm{c}}=0$ ). Moreover, for the present purpose it is sufficient to retain only terms of first order in the noise; $m_{1}$ is itself of first order since the noisefree part of the correlation function is symmetrical, so we obtain the approximation

$\delta \cong K_{0} m_{1}$

where $K_{0}$ is given by (A1) and the subscript 0 again indicates that noise contributions have been omitted. The variance $\sigma_{\mathrm{P}}^{2}$ of the correlation-peak position is obtained now as the statistical average of $\delta^{2}$ :

$\sigma_{\mathrm{P}}^{2} \equiv\left\langle\delta^{2}\right\rangle=\frac{K_{0}^{2}}{C^{2}} \sum_{n=0}^{N-1} g^{2}(n, p)\left[\sigma_{\mathrm{S}}^{2}(n)+\sigma_{\mathrm{T}}^{2}(n)\right]$

where we used $\left\langle N_{\mathrm{S}, \mathrm{T}}(n)\right\rangle=0$ and defined

$$
\begin{aligned}
g(n, p) & =\sum_{j=-p}^{p} j S_{0}(n \oplus j) \\
\sigma_{\mathrm{S}, \mathrm{T}}(n) & =\left\langle N_{\mathrm{S}, \mathrm{T}}^{2}(n)\right\rangle .
\end{aligned}
$$

By its definition, the random error $\epsilon$ in Sect. 5 must be identified with $\sigma_{\mathrm{P}}$.

With spectra given by Eq. (A3), both the error on the position of the cross-correlation maximum and the antisymmetric part of the correlation function originate from the noise only. Pre-eminently in this case, any relation between those two should be revealed most clearly, at least through their statistical average. We therefore calculated the average of ${\sigma_{\mathrm{a}}}^{2}$ defined in Eq. (11), applied with $\delta=0$ since there is no intrinsic shift:

$\left\langle\sigma_{\mathrm{a}}{ }^{2}\right\rangle=\frac{1}{C^{2}} \sum_{n=0}^{N-1} h(n)\left[\sigma_{\mathrm{S}}^{2}(n)+\sigma_{\mathrm{T}}^{2}(n)\right]$

where

$h(n)=\frac{1}{2 N} \sum_{i=0}^{N / 2-1}\left[S_{0}(n \oplus i)-S_{0}(n \ominus i)\right]^{2}$.

\section{References}

Baranne A., Queloz D., Mayor M., et al., 1996, A\&AS 119, 373 Brown T.M., 1989, CCD's in Astronomy, Jacoby G.H. (ed.), ASP Conf. Ser. 8, 335

Butler R.P., Marcy G.W., Williams E., et al., 1996, PASP 108, 500

Collins II G.W., Truax R.J., 1995, ApJ 439, 860

Connes P., 1985, Ap\&SS 110, 211

David M., Verschueren W., 1995, A\&AS 111, 183

Fellgett P., 1953, Opt. Acta 2, No. 9, 112

Furenlid I., Furenlid L., 1990, PASP 102, 592

Gray D.F., 1976, in: The Observation and Analysis of Stellar Photospheres. J. Wiley and Sons, Chapter 17

Griffin R., 1967, ApJ 48, L48

Kurtz M.J., Mink D.J., 1998, PASP 110, 934

Kurtz M.J., Mink D.J., Wyatt W.F., et al., 1992, Astronomical Data Analysis Software and Systems I, Worrall D.M., Biemesderfer C. \& Barnes J. (eds.) ASP Conf. Ser. 25, 432

Latham D.W., 1992, IAU Colloq. 135 Complementary Approaches to Binary and Multiple Star Research, McAlister H. \& Hartkopf W. (eds.) ASP Conf. Ser. 32, 110 Merline W.J., 1985, IAU Colloq. 88 Stellar Radial Velocities, Davis Philip A.G. \& Latham D.W. (eds.). L. Davis Press, p. 87

Murdoch K., Hearnshaw J.B., 1991, Ap\&SS 186, 137

Peterson R.C., 1983, ApJ 275, 737

Peterson R.C., Carney B.W., Latham D.W., 1984, ApJ 279, 237

Scarfe C.D., Batten A.H., Fletcher J.M., 1990, Publ. Dom. Astr. Obs. 18, No. 2, p. 21

Tonry J., Davis M., 1979, AJ 84, 1511

Verschueren W., 1991, Ph. D. Thesis. Free Univ. of Brussels (VUB), Belgium

Verschueren W., 1995, The Standard Star Newsletter 18, 6 C. Corbally (ed.)

Verschueren W., David M., Vrancken M., 1999a, IAU Colloq. 170 Precise Stellar Radial Velocities, ASP Conf. Ser. Hearnshaw J.B. \& Scarfe C.D. (eds.) (in press)

Verschueren W., David M., Griffin R., 1999b, A\&A (submitted)

Vrancken M., Hensberge H., David M., Verschueren W., 1997, A\&A 320,878

Woodward P.M., 1953, Probability and Information Theory, with Applications to Radar. Pergamon Press, London

Woodward P.M., Davies I.L., 1950, Phil. Mag. 41, 1001 